\title{
Engineering the spatial confinement of exciton polaritons in semiconductors
}

\author{
R. Idrissi Kaitouni, ${ }^{1}$ O. El Daïf, ${ }^{1}$ A. Baas, ${ }^{1}$ M. Richard, ${ }^{1}$ T. Paraiso, ${ }^{1}$ P. Lugan,${ }^{1,2}$ T. Guillet,,${ }^{3}$ F. Morier-Genoud,${ }^{1}$ \\ J. D. Ganière, ${ }^{1}$ J. L. Staehli, ${ }^{1}$ V. Savona, ${ }^{2, *}$ and B. Deveaud ${ }^{1}$ \\ ${ }^{1}$ Institute of Quantum Electronics and Photonics, Ecole Polytechnique Fédérale de Lausanne EPFL, CH-1015 Lausanne, Switzerland \\ ${ }^{2}$ Institute of Theoretical Physics, Ecole Polytechnique Fédérale de Lausanne EPFL, CH-1015 Lausanne, Switzerland \\ ${ }^{3}$ Groupe d'Etude des Semiconducteurs (GES), Université de Montpellier II, Place Eugène Bataillon, F-34095 Montpellier, France
}

(Received 14 August 2006; revised manuscript received 7 September 2006; published 16 October 2006)

\begin{abstract}
We demonstrate three-dimensional spatial confinement of exciton-polaritons in a semiconductor microcavity. Polaritons are confined within a micron-sized region of slightly larger cavity thickness, called mesa, through lateral trapping of their photon component. This results in a shallow potential well that allows the simultaneous existence of extended states above the barrier. Photoluminescence spectra were measured as a function of either the emission angle or the position on the sample. Striking signatures of confined states of lower and upper polaritons, together with the corresponding extended states at higher energy, were found. In particular, the confined states appear only within the mesa region, and are characterized by a discrete energy spectrum and a broad angular pattern. A theoretical model of polariton states, based on a realistic description of the confined photon modes, supports our observations.
\end{abstract}

DOI: 10.1103/PhysRevB.74.155311

PACS number(s): 71.36.+c, 42.65.-k, 71.35.Lk

\section{INTRODUCTION}

Most of the major advances in semiconductor physics and technology over the last 30 years originated from quantum confinement of elementary excitations along one, two, or three spatial dimensions ${ }^{1-4}$ and to the improvement of their coupling to the electromagnetic field. In this context, quantum $\operatorname{dots}^{3}$ represent the prototypical system. They are often called "macroatoms" as they allow quasi-zero-dimensional confinement of electronic states and display a discrete spectrum of energy levels. Quantum dots are usually fabricated by a spontaneous formation process, resulting in a broad distribution of sizes and shapes. ${ }^{3}$ This in turn limits the control over the energy-level structure and makes single-dot applications a challenging task.

As an alternative to electron-hole pairs in quantum dots, confined states of other kinds of excitations in solids can be engineered. To this purpose, two-dimensional polaritons in planar semiconductor microcavities ${ }^{6-8}(\mathrm{MCs})$ are particularly suited. In MCs, the photon part of the polariton is provided by the optical modes of a Fabry-Pérot planar semiconductor resonator, which are resonant with the exciton level of an embedded semiconductor quantum well $(\mathrm{QW})$. The dependence of polariton energy on its in-plane momentum has a quadratic behavior, reminding of a massive particle, with an effective mass typically of the order of $10^{-5}$ times the free electron mass. ${ }^{9}$ Hence a sizeable spacing of energy levels is expected already when the confinement extends over a few microns-a quite unique situation in a semiconductor artificial structure that makes fabrication, positioning, and optical addressing much easier than for other nanostructured systems. Owing to their peculiar nature of weakly interacting bosonic quasiparticles, confined polaritons would be an optimal system for a wide range of fundamental and applied studies. Polariton parametric processes ${ }^{10,11}$ could be exploited for producing confined polaritons in quantum states displaying nonclassical properties like quantum correlations and entanglement. ${ }^{12,13}$ This, joined to the ease of integration, optical manipulation, and readout, could be the premise for a novel kind of quantum information device. Moreover, the discrete spectrum is the key feature ${ }^{14}$ to overcome the effect of quantum fluctuations that dominate a two-dimensional interacting Bose gas, and opens the way to the observation of collective many-body effects. ${ }^{15-17}$

Attempts to produce spatially confined polariton states, in the past, have been made by etching micropillars ${ }^{18,19}$ of a few $\mu \mathrm{m}$ in diameter from an initially planar MC. ${ }^{20-23}$ These structures have in general proved able of producing lateral confinement of polariton modes. However, they tend to display strong coupling only in the limit of very small diameter, ${ }^{20,21}$ typically in the 1 to $2 \mu \mathrm{m}$ range. Sometimes the spectral signature of the upper polariton-needed as a proof of the formation of polaritons as normal modes of the linear exciton-photon coupling - is completely absent. ${ }^{22}$ In a very recent work, ${ }^{24}$ we described a new paradigm of devices able of producing laterally confined polariton states in a MC. A spectral analysis has revealed a series of sharp emission lines that display avoided level crossing when varying the exciton-cavity detuning. In spite of this promising result, however, a direct experimental characterization of the simultaneous spatial confinement of upper and lower polariton modes is still needed.

In this work we present conclusive direct evidence of the spatial confinement of MC polaritons in a three-dimensional trap based on this approach. The trap is obtained by a shallow pattern etched on top of the cavity layer of a semiconductor microcavity, before growing the top mirror. ${ }^{24}$ One of the most relevant features of this technique is that the confinement potential has shallow energy barriers. Hence both confined states inside the trap and extended states above the barrier are present. The present analysis is carried out by means of both angle-resolved and spatially resolved photoluminescence (PL) spectroscopy.

The sample under inversigation ${ }^{24}$ contains nominally circular mesas of three different diameters, at varying excitoncavity detuning. We perform PL spectra under nonresonant excitation, and resolve the PL emission either in real or in 


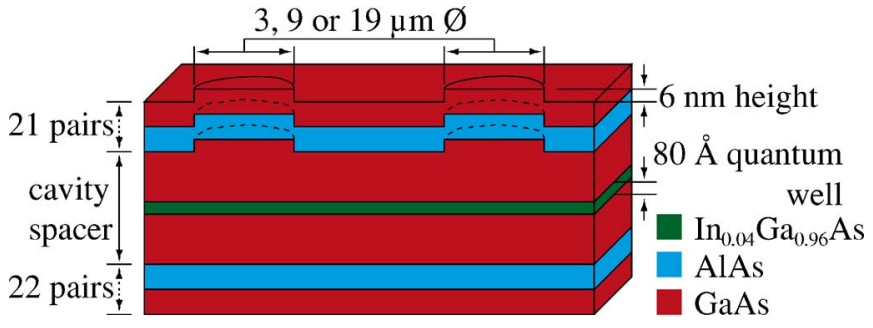

FIG. 1. (Color online) Sketch of the sample (cross-section view). For clarity, the various lengths are not represented to scale. In particular, the mesa pattern has a height $\Delta L=6 \mathrm{~nm}$, as indicated, while the cavity spacer is approximately $230 \mathrm{~nm}$ thick.

reciprocal space. All PL spectra display clear signatures of laterally confined upper and lower polariton states, and of correspondingly extended polariton states above the barrier of the confinement potential. A theoretical model of the polariton states in the mesa is developed for the interpretation of the experimental data. The model is based on the solution of Maxwell equations for the electromagnetic field, assumed as scalar, in a perturbed planar geometry. The photon modes and the exciton band in the QW are then used in a linear coupling Hamiltonian to obtain the polariton states. When the nominal structural parameters of the mesas are used (mesa height and shape), the theory reproduces the measured spectra very satisfactorily, proving the effectivenenss of the fabrication technique.

In Sec. II, we illustrate the technique for obtaining a lateral confining trap for polaritons, and we describe the sample under investigation. In Sec. III, the PL measurements are presented. Section IV presents a comprehensive description of the theoretical model. We discuss the results in Sec. V. Finally, Sec. VI contains our conclusions and outlook.

\section{PATTERNED MESAS IN MICROCAVITIES}

Let us assume a semiconductor MC made of a $\lambda_{c}$-thick spacer sandwiched between two distributed Bragg reflectors (DBRs). Following the scheme that we recently proposed, ${ }^{24}$ in order to create a polariton trap the thickness of the MC spacer is made slightly larger by anamount $\Delta L$ within a limited region of the MC plane that we call mesa, as sketched in Fig. 1. This local variation is obtained by etching the mesa pattern on top of the cavity spacer and then growing the top DBR. To an increased cavity thickness corresponds a local decrease of the resonant photon-mode energy. The spatial pattern then acts as a two-dimensional confining potential for the photon mode. As the photon couples to the exciton, forming a polariton mode, the mesa then becomes a polariton trap. We point out that only the thickness of the MC spacer is varied, while the optical thickness of the DBR layers is assumed everywhere equal to $\lambda_{c} / 4$. Therefore within the mesa the DBRs are slightly detuned with respect to the spacer thickness. This makes fabrication easier and implies a less pronounced dependence of the local mode energy on $\Delta L$. In fact, ${ }^{8}$ the lowest MC resonant fequency is obtained as the solution of $\left(n_{c} / c\right)\left[\left(\omega-\omega_{c}\right) L_{c}+\left(\omega-\omega_{m}\right) L_{D B R}\right]=0$, where $L_{c}$ is the local cavity thickness, $\omega_{c}$ and $\omega_{m}$ are, respectively, the resonant frequency of the cavity spacer and of the DBRs, and $L_{D B R}=\left(\lambda_{c} / 2\right) n_{1} n_{2} /\left[n_{1}\left(n_{1}-n_{2}\right)\right]$ is the field penetration length in the DBRs, expressed in terms of the two DBR refraction indices $n_{1}>n_{2}$. By replacing $L_{c}=\lambda_{c}+\Delta L, \omega_{m}=\left(c / n_{c}\right) 2 \pi / \lambda_{c}$, and $\omega_{c}=\left(c / n_{c}\right) 2 \pi /\left(\lambda_{c}+\Delta L\right)$, we obtain $\Delta \omega_{c}=-\omega_{m} \Delta L /\left(\lambda_{c}\right.$ $\left.+L_{D B R}\right)$. For ideal DBRs tuned to the spacer frequency, instead, $\Delta \omega_{c}=-\omega_{m} \Delta L / \lambda_{c}$. This weaker dependence on $\Delta L$ has thus the further advantage of allowing finer control over the energy offset of shallow mesas, for which a $\Delta L$ of only a few $\mathrm{nm}$ must be obtained in the fabrication process. For typical GaAs/AlAs DBRs, $L_{D B R} \simeq 3 \lambda_{c}$. Given $\Delta L=6 \mathrm{~nm}$ in Ref. 24, the previous expression predicts a mesa energy offset of $\hbar \Delta \omega_{c}=-9 \mathrm{meV}$, in agreement with the optical characterization of the sample. The finite height of the barrier in the confining potential results in both spatially confined photon modes and a continuum of extended photon modes at energies above the barrier. The polaritons resulting from the strong coupling of these photon modes with the quantum well exciton will also display a mixed spectrum containing both confined and extended states. In addition to an easier fabrication approach, this kind of structure features a considerable difference with respect to micropillars, ${ }^{18,19,22}$ where photon confinement is obtained by etching the whole cavity body, thus resulting exclusively in confined modes.

The sample studied in this work, ${ }^{24}$ sketched in Fig. 1, is a $\lambda_{c}$-thick GaAs semiconductor MC sandwiched between AlAs/GaAs DBRs made of 21 (top) and 22 (bottom) double $\lambda_{c} / 4$ layers. Embedded at the MC center is a single $8 \mathrm{~nm}$ $\mathrm{In}_{0.04} \mathrm{Ga}_{0.96}$ As quantum well, characterized by a sharp exciton resonance at $1.484 \mathrm{eV}$. A wedge of $2.4 \mathrm{meV} / \mathrm{mm}$ of the microcavity wafer allows varying the cavity detuning across the exciton resonance. Before growing the top mirror, a pattern of $6 \mathrm{~nm}$ height has been chemically etched on the cavity spacer using a photolithography mask. The pattern shape and height is preserved throughout the growth up to the Bragg mirror top surface, as measurements by means of an atomicforce microscope show. Furthermore, these measurements suggest that the actual structures are not perfectly circular and their diameters slightly differ from their nominal values. Circular mesas with nominal diameter of 3, 9, and $19 \mu \mathrm{m}$ were patterned. The mesas are regularly spaced along the direction of the cavity wedge, so that mesas with varying exciton-cavity detuning could be achieved. All experiments were carried out at $T=4 \mathrm{~K}$ and consisted in PL measurements in the linear regime under pulsed off-resonance excitation at $760 \mathrm{~nm}$, within the exciton continuum band. The sample was placed in the focal plane of a microscope objective. A lens placed between the microscope objective and the mochromator allows, depending on its position, to image either the Fourier plane or the real-space emission pattern. The monochromator slit selects a narrow stripe of the solid angle, which is then dispersed inside the monocromator and detected by a charge coupled device (CCD) camera. In this way, thanks to the cylindrical symmetry, the PL spectrumresolved either in momentum or in real-pace depending on the chosen configuration-is directly displayed by the CCD. The excitation spot cross section had a Gaussian profile. For momentum-resolved measurements, a Gauss spotsize of $3 \mu \mathrm{m}$ was used in order to span a large enough momentum range. For real-space measurements, on the other hand, the 
Gauss spot size was $25 \mu \mathrm{m}$, which excites an area larger than the mesa and makes it possible to observe emission both from spatially confined and extended states. As the distance between mesas is $125 \mu \mathrm{m}$, in each case a single mesa was excited.

\section{PHOTOLUMINESCENCE MEASUREMENTS}

When PL is measured from unprocessed regions far away from the mesas, a typical spectrum of polaritons in a planar $\mathrm{MC}$ is observed. The measured vacuum field Rabi splitting is $3.8 \mathrm{meV}$, with the extrapolated cavity-mode and exciton linewidths being 200 and $500 \mu \mathrm{eV}$, respectively. Large patterned regions $(250 \mu \mathrm{m})^{24}$ reveal a similar polariton dispersion with a cavity mode energy $9 \mathrm{meV}$ lower than in unprocessed regions. This value is consistent with a $\Delta L=6 \mathrm{~nm}$ thickness variation from mesa to barrier, already pointed out. When the excitation spot is focused on one mesa, the PL spectrum shows discrete narrow lines, in addition to a weak signature of the extended polariton states identical to that measured in the unprocessed regions of the sample.

Real-space images give direct visual proof of polariton confinement. The deviations of the actual structure from a perfectly circular trap, however, result in confined states with slightly irregular shapes. Since only a narrow stripe is selected from the plane for imaging, the real-space information might not be directly comparable to the result of a model of a perfectly circular trap. A more regular pattern is obtained by measuring the amplitude of the polariton wave function in momentum-space. We thus begin with the analysis of momentum-resolved spectra. As the polariton in-plane momentum is related to the emission angle from the normal axis, via the relation $\mathbf{k}=\sqrt{\epsilon_{0}}(\omega / c) \sin (\theta)$, we report directly the angle-resolved spectral pattern. The measured patterns for 3, 9, and $19 \mu \mathrm{m}$ diameter mesas are displayed in Figs. 2(a)-2(c). The dispersion of the extended polariton (barely visible in the $19 \mu \mathrm{m}$ mesa) is highlighted by dashed lines. We estimate a positive detuning of $6.4 \mathrm{meV}$ between the extended cavity mode and the exciton. The strongest spectral features in the three images appear immediately below and above the lower extended polariton mode. In particular, the $3 \mu \mathrm{m}$ mesa shows few discrete lines extending over broad angular regions, with energy spacings in the $\mathrm{meV}$ range. The linewidth of the lowest confined polariton level reaches $70 \mu \mathrm{eV}$ in this mesa, yielding a $Q$-factor as high as 21000 . This linewidth should be dominated by the polariton radiative rate, resulting in a polariton lifetime of $10 \mathrm{ps}$, still shorter than any other dephasing mechanism expected in this system at low temperature and density. The fact that the confined polariton linewidth is smaller than one-half of the photon-mode linewidth in the planar cavity suggests that this latter is affected by some inhomogeneous broadening due to long-range cavity thickness fluctuations. Compared to the $3 \mu \mathrm{m}$ mesa, the $9 \mu \mathrm{m}$ one shows a larger number of more closely spaced spectral lines, with a smaller angular spread. Finally, in the $19 \mu \mathrm{m}$ mesa these features approach a quasicontinuous spectrum, while the angular spread is still smaller.

Figures 3(a)-3(c) show three PL spectral patterns in realspace, for the 3, 9, and $19 \mu \mathrm{m}$ mesa, respectively. These
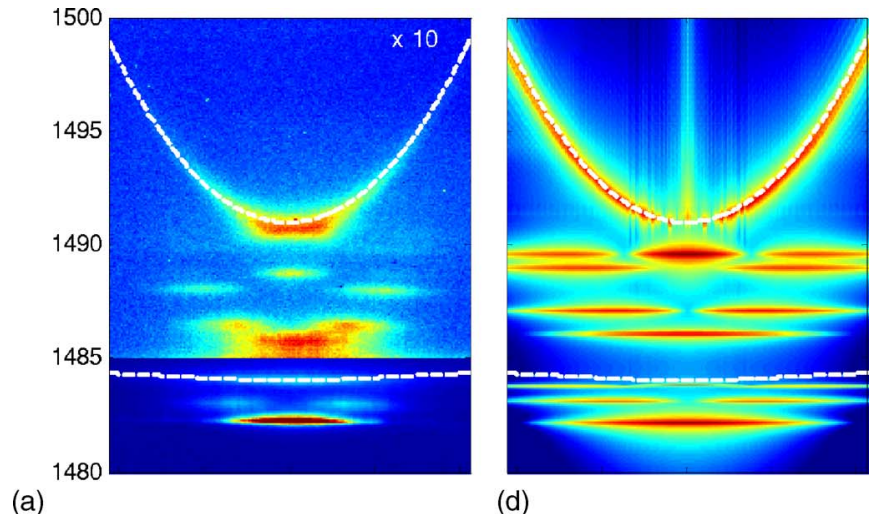

(d)
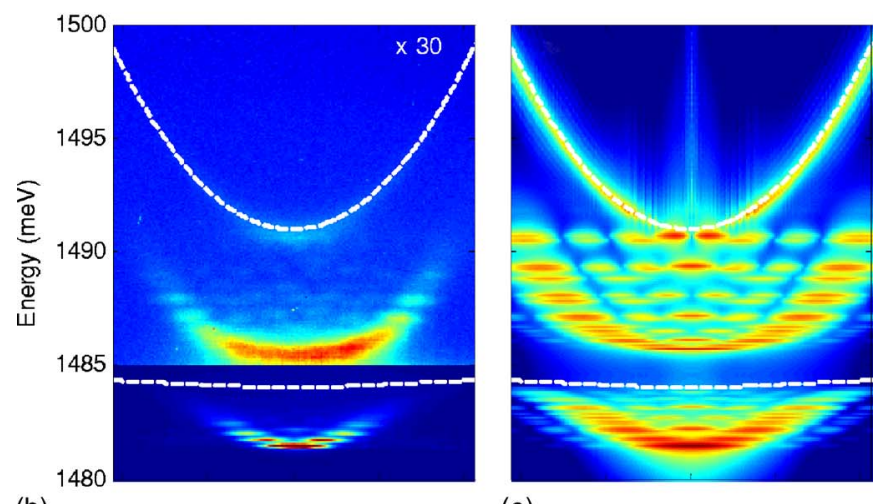

(b)

(e)

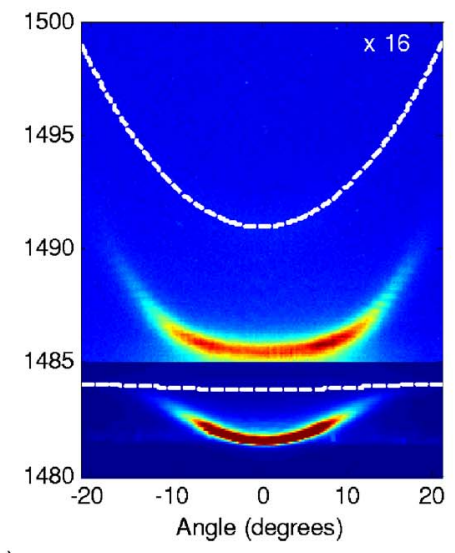

(c)

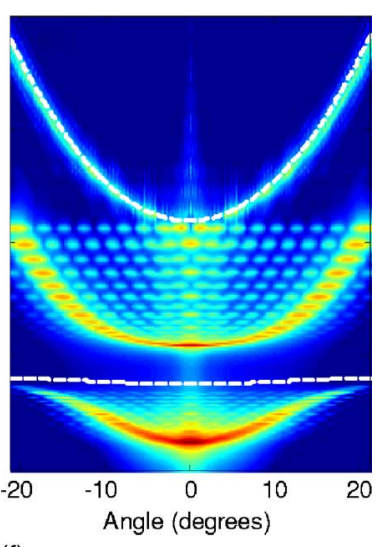

(f)

FIG. 2. (Color online) (Left) Measured polariton PL intensity (linear color scale from blue to red) as a function of energy and emission angle for the $3 \mu \mathrm{m}$ (a), $9 \mu \mathrm{m}$ (b), and $19 \mu \mathrm{m}$ (c) mesa. For clarity, the intensity above $1485 \mathrm{meV}$ is multiplied by a constant factor, as indicated. Dashed: dispersion of the extended polariton modes, computed from a coupled oscillator model. (Right) Intensity plot of the simulated polariton spectral density for the $3 \mu \mathrm{m}$ (d), $9 \mu \mathrm{m}$ (e), and $19 \mu \mathrm{m}$ (f) mesa (color log-scale, 4 decades from blue to red).

spectra were obtained by excitation with a spot diameter larger than the mesas. The spectrum for the $3 \mu \mathrm{m}$ mesa in Fig. 3(a) was taken at a detuning comparable to that of Fig. 2(a), whereas for the two other mesas in Figs. 3(b) and 3(c) a detuning close to zero for the extended modes was chosen. Again, as for the momentum-space images, confined and extended spectral features of both upper and lower polaritons 

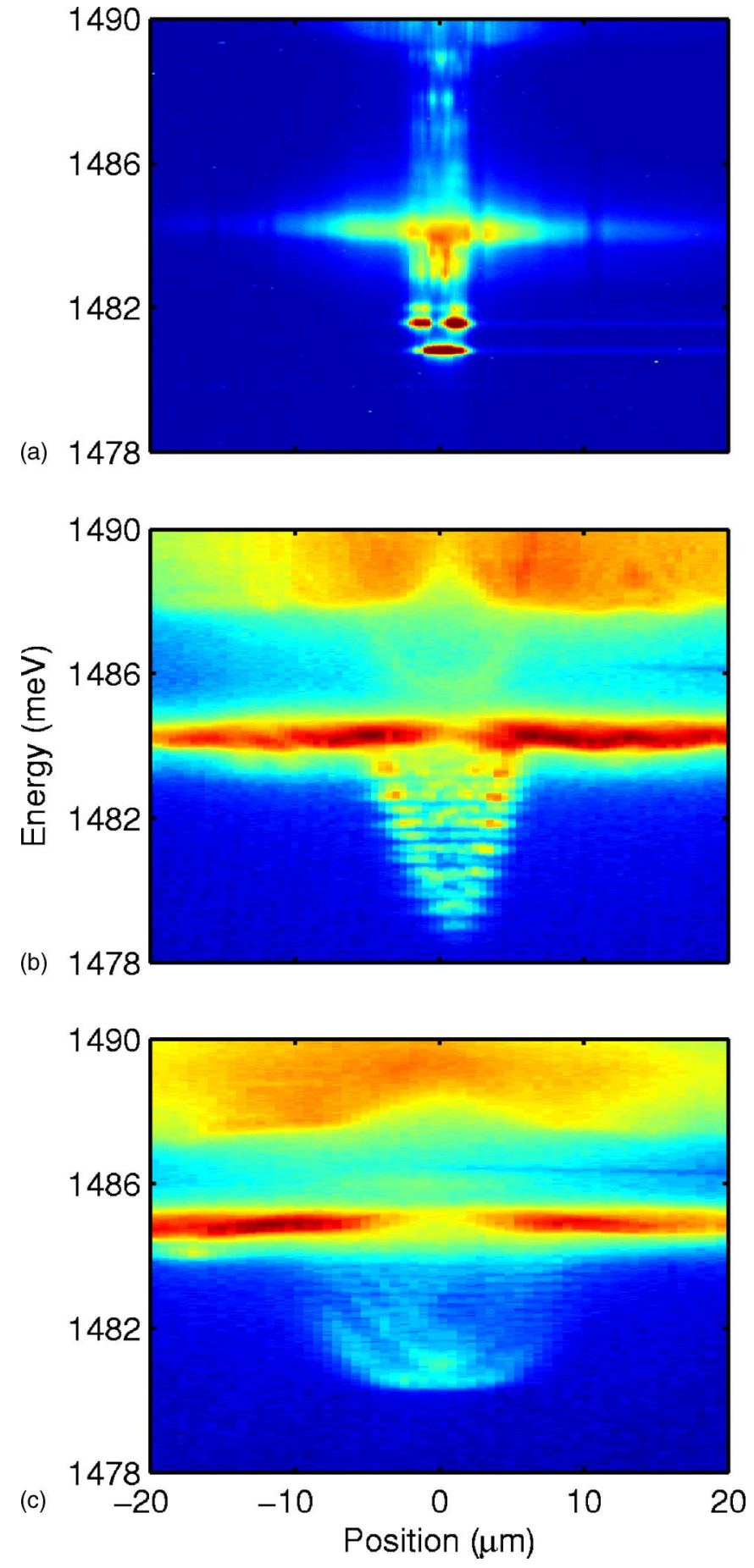

FIG. 3. (Color online) Measured polariton PL intensity (color log-scale varying over 1.4 decades from blue to red) as a function of energy and position on the sample, for the $3 \mu \mathrm{m}$ (a), $9 \mu \mathrm{m}$ (b), and $19 \mu \mathrm{m}$ (c) mesa. The horizontal lines to the right of the lowest levels in (a), as well as the faint darker vertical stripes, are experimental artifacts.

are clearly visible. Notice in particular the confined upper polariton, that has a weaker PL signal as polaritons tend to relax towards the lowest energy states of the trap, but is still visible above $1486 \mathrm{meV}$ in the three spectra.
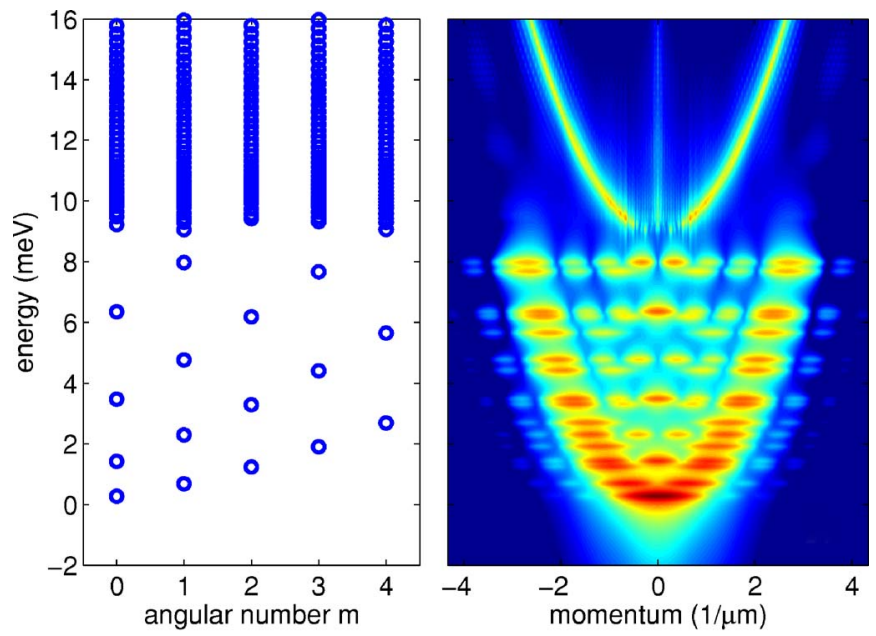

(a)

(b)

FIG. 4. (Color online) (a) Computed energy eigenvalues for the photon modes of a circular mesa $\left(D=8.6 \mu \mathrm{m}, \hbar \Delta \omega_{c}=-9 \mathrm{meV}\right)$. (b) Intensity plot of the photon spectral density (log scale, 4 decades from blue to red).

\section{THEORY}

In order to support the measured data, we develop a theoretical model of trapped polaritons. For a shallow mesa of lateral extension larger than the optical wavelength, we can safely assume that the electromagnetic modes at in-plane position $\boldsymbol{\rho}$ are locally equivalent to those of a planar microcavity:

$$
E(\mathbf{r})=E(\boldsymbol{\rho}) \exp \left[i k_{z}(\boldsymbol{\rho}) z\right],
$$

where $k_{z}(\boldsymbol{\rho})$ is piecewise constant. For simplicity, here we assume a scalar electric field. If one is interested in the polarization properties of light, then the model must be extended in order to include the vector nature of the field and the selection rules for the coupling with the different components of the exciton interband polarization. Neglecting border effects at the mesa contour, Maxwell equations give

$$
\nabla_{\rho}^{2} E(\boldsymbol{\rho})+\left(\frac{\omega^{2}}{c^{2}} \epsilon_{0}-k_{z}^{2}(\boldsymbol{\rho})\right) E(\boldsymbol{\rho})=0,
$$

where $\epsilon_{0}$ is the background dielectric constant of the MC spacer layer. Outside the mesa, the MC resonance is $k_{z}$ $=2 \pi / \lambda_{c}$. Inside the mesa we can relate the offset $\Delta k_{z}$ to the energy offset $\Delta \omega_{c}$, as $\Delta k_{z}=\sqrt{\epsilon_{0}} \Delta \omega_{c} / c$. Equation (2) is then solved in cylindrical coordinates, assuming a circular mesa of diameter $D$. The eigenmodes are therefore expressed as $E(\boldsymbol{\rho})=U_{n m}(\rho) \exp (i m \phi)$, where $n=0,1, \ldots$ and $m=-n, \ldots, n$ are the radial and angular mode numbers, respectively. The mode eigenenergies and the corresponding energymomentum spectral function are plotted in Figs. 4(a) and 4(b) for a mesa of diameter $D=8.6 \mu \mathrm{m}$, as an example. A discrete energy spectrum appears, whose modes show a flat extended signature in momentum space, reflecting their spatial confinement. For energies above the $9 \mathrm{meV}$ barrier, we find a continuum of states whose energy-momentum signature practically coincides to that of a planar MC. This result 
already suggests that the structure is able to confine photons in the three spatial directions.

We similarly express the exciton center-of-mass wave function in terms of Bessel functions of the first kind, $\psi_{n m}(\boldsymbol{\rho})=N_{n m} J_{m}\left(\kappa_{n m} \rho\right) \exp (i m \phi)$, where $\kappa_{n m}$ are the exciton eigen-momenta and $N_{n m}$ is a normalization constant. These are the modes of a free particle, as the exciton motion is not affected by the mesa structure. By introducing Bose operators $\hat{A}_{n m}$ and $\hat{B}_{n m}$ for photon and exciton modes, respectively, the linear exciton-photon Hamiltonian can be finally expressed in second quantization as

$$
\begin{aligned}
H= & \sum_{m}\left[\sum_{n} \hbar \omega_{n m}^{(p h)} \hat{A}_{n m}^{\dagger} \hat{A}_{n m}+\sum_{n} \hbar \omega_{n m}^{(e x c)} \hat{B}_{n m}^{\dagger} \hat{B}_{n m}\right. \\
& \left.+\left(\sum_{n n^{\prime}} \frac{\hbar \Omega_{n n^{\prime}}^{(m)}}{2} \hat{A}_{n m}^{\dagger} \hat{B}_{n^{\prime} m}+\text { H.c. }\right)\right]
\end{aligned}
$$

where $\omega_{m n}^{(p h)}$ and $\omega_{m n}^{(e x c)}$ are the eigenenergies of the photon and of the (free) exciton modes. As required by symmetry, the angular number $m$ is conserved in the coupling. The energies $\hbar \Omega_{n n^{\prime}}^{(m)}$ are expressed in terms of the Rabi splitting of the planar cavity $\hbar \Omega_{R}$ and of exciton-photon overlap integrals

$$
\Omega_{n n^{\prime}}^{(m)}=2 \pi \Omega_{R} \int d \rho \rho U_{n m}^{*}(\rho) N_{n^{\prime} m} J_{m}\left(\kappa_{n^{\prime} m} \rho\right)
$$

Here, the vacuum-field Rabi splitting of the planar MC is assumed as an input parameter and taken as $\hbar \Omega_{R}=3.8 \mathrm{meV}$, according to the measured polariton dispersion.

For a planar geometry, momentum conservation implies a one-to-one coupling between exciton and photon modes. Here, on the contrary, no selection rule on the radial quantum number $n$ exists. For the numerical solution we therefore choose to retain only a finite number of cavity modes $N_{c}$ and exciton modes $N_{x}$ for each value of $m$. The resulting polariton modes are obtained by numerical diagonalization of the $\left(N_{c}+N_{x}\right) \times\left(N_{c}+N_{x}\right)$ matrix obtained from the Hamiltonian (3). Polariton eigenvalues obtained for $D=8.6 \mu \mathrm{m}$ are plotted in Fig. 5(a). A detuning of $\Delta=+6.5 \mathrm{meV}$ of the planar cavity mode with respect to the exciton was assumed in order to bring the lowest confined photon modes in resonance with the bare exciton. The result in Fig. 5(a) brings clear evidence of a discrete energy spectrum, followed by a continuous spectrum at higher energy, both for the lower and for the upper polariton. The energy spacings in this case are of the order of $1 \mathrm{meV}$. The polariton operators obtained from the diagonalization of Eq. (3) are expressed as $\hat{P}_{n m}$ $=\Sigma_{n^{\prime}}\left(X_{n m}^{n^{\prime}} \hat{B}_{n^{\prime} m}+W_{n m}^{n^{\prime}} \hat{A}_{n^{\prime} m}\right)$. Each polariton mode exhibits an angular emission pattern according to its photon component in momentum space, defined as $I_{n m}(\mathbf{k})=\left|\left\langle\mathbf{k}\left|\hat{P}_{n m}^{\dagger}\right| 0\right\rangle\right|^{2}$, that is easily computed from the model. By assuming for each mode a Lorentzian energy spectrum, we can finally compute an energy-momentum spectral function, as shown in Fig. 5(b). In the discrete part of the spectrum, polariton modes present a flat, broad energy-momentum signature, which corresponds to the Fourier transform of spatially confined states.

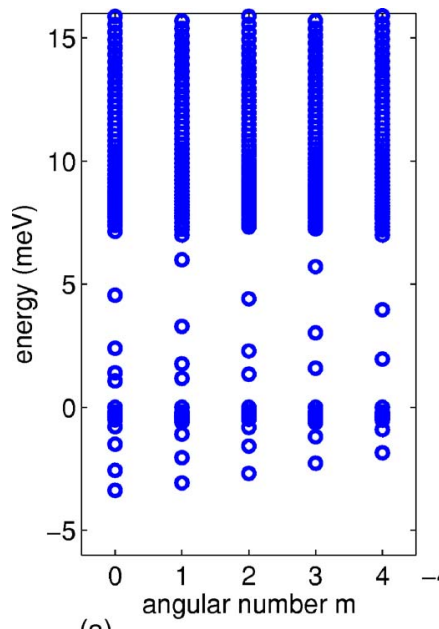

(a)

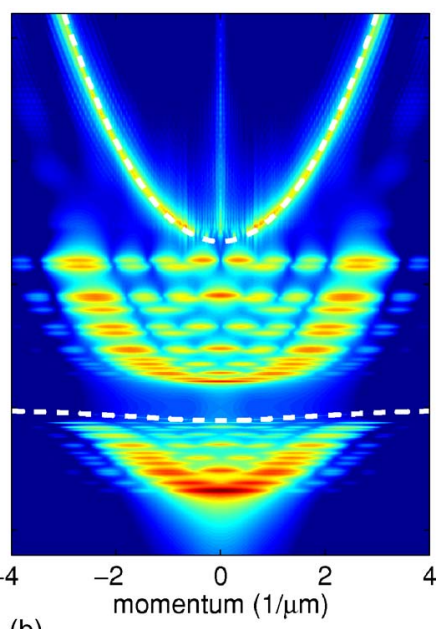

(b)
FIG. 5. (Color online) (a) Computed polariton eigenvalues for $D=8.6 \mu \mathrm{m}$. (b) Intensity plot of the polariton energy-momentum spectral function for $D=8.6 \mu \mathrm{m}$ (log scale, 4 decades from blue to red). The dashed lines indicate the bare polariton dispersion of the planar MC corresponding to the mesa barrier.

The continuous part of the spectrum, on the other hand, simply corresponds to the dispersion of quasifree twodimensional polaritons. These are the scattering states above the finite energy barriers of the potential formed by the mesa. Correspondingly, the energy-momentum dispersion is welldefined, with a negligible broadening in $k$-space. For the present detuning, the spectrum of the lowest extended polariton is strongly suppressed, as these are almost fully excitonlike with vanishing photon fraction. We point out that the discrete modes in Fig. 5(b) follow a pattern that mimicks the energy-momentum dispersion of two-dimensional (2D) polaritons. For diameters larger than $20 \mu \mathrm{m}$, the simulation results in a spectrum practically identical to that of $2 \mathrm{D}$ polaritons in a MC of thickness $\lambda_{c}+\Delta L$.

\section{DISCUSSION}

The experimental data presented in Sec. III provide clear evidence for the coexistence of confined and extended polariton states in our patterned mesas. Here, we discuss the outcome of the measurements, both angle- and space-resolved, and compare them to the result of the simulations.

The angle-resolved PL spectra in Fig. 2 show, as a general trend, a narrowing of the angular pattern at fixed energy and a decrease of the energy spacing as the mesa diameter increases. This trend is observed for any value of the excitoncavity detuning. Spatial confinement explains in a natural way these observations. Indeed, confinement induces a discrete energy spectrum and localization of the wave functions in real space, which in turn produces flat extended features in reciprocal space. The mesas therefore act like spatial traps. Still, it must be verified that the measured spectral lines actually originate from polariton states, namely normal modes of the linear coupling between exciton and cavity photons. For extended polaritons, the evidence of this strong coupling regime is usually given by the level anticrossing between the 
two polariton branches in the energy-momentum dispersion. For confined states, the discrete energy spectrum makes level anticrossing more difficult to characterize. In the case of the 9 and $19 \mu \mathrm{m}$ mesas in Figs. 2(b) and 2(c), however, the discrete levels form a pattern displaying a distinct level anticrossing at approximately $15^{\circ}$, with a vacuum field Rabi splitting close to the $3.8 \mathrm{meV}$ measured for the extended polaritons. This is a clear proof of strong coupling. For the $3 \mu \mathrm{m}$ mesa, this feature is more difficult to characterize. However, we remark in Fig. 2(a) that the two levels at 1482.5 and $1483 \mathrm{meV}$, thus below the bare exciton energy, display the same angular pattern as the two levels lying above the bare exciton energy at 1485.5 and $1486 \mathrm{meV}$. This clearly gives evidence to the fact that they are, respectively, lower and upper confined polariton states. This analysis proves that the strong coupling is preserved by spatial confinement and the species emitting are indeed mixed excitonphoton modes.

The model described in the previous section allows one to simulate the shape of the measured spectra. For these simulations, circular mesas were assumed. The values of the diameter $D$ and the detuning $\Delta$ used in the simulations were fitted to the experimental data. For the 3, 9, and $19 \mu \mathrm{m}$ mesa we obtained, respectively, $D=3.46,8.6$, and $20.0 \mu \mathrm{m}$, and $\Delta=5.9,6.8$, and $7.1 \mathrm{meV}$. For the remaining parameters, the nominal values of the sample were used. Figures 2(d)-2(f) display the simulated polariton spectral density for the three different mesas. We point out that the relative spectral intensities in the measured PL bear additional information on the polariton state populations that cannot be accounted for in the simulated spectral density. A slight discrepancy in the energies of the smallest mesa is probably due to its not perfectly circular shape. In general, however the model faithfully reproduces both the energy position and angular extension of the various spectral features. This is an additional proof that the mesa structures are efficient traps for microcavity polaritons. Having used directly the nominal parameters of the samples for the simulation further suggests that these structures operate in a very effective way as polariton traps. Spurious effects, other than a slight deviation from a perfectly circular shape, are practically absent because, as opposite to micropillars, the fabrication technique is much less invasive.

Better insight into the polariton confinement mechanism can be gained from the real-space measurements, displayed in Figs. 3(a)-3(c) for the three mesas, respectively. Here the excitation spot had a Gauss diameter of $25 \mu \mathrm{m}$, thus exciting a much wider area than the mesa. As a result, PL from the spatially extended states is dominant in the 9 and $19 \mu \mathrm{m}$ mesas, while the larger positive detuning in the case of the $3 \mu \mathrm{m}$ mesa still allows a good relaxation to the trapped states. In general, however, confined upper and lower polaritons are clearly visible in the three spectra. Confined levels of the lower polariton, in particular, show a pattern following the spatial dependence of the polariton wave function. Some of these spectral features are characterized by a marked asymmetry with respect to the left-right inversion that can be partly understood in terms of the deviation of the mesa shape from a perfect cylinder. We also notice the irregular intensity

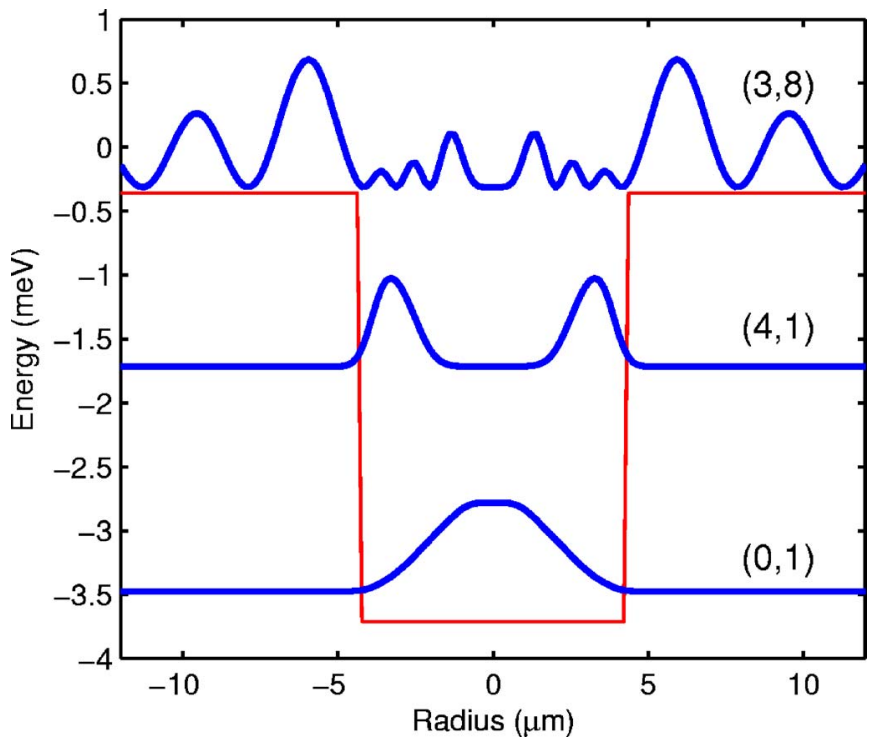

FIG. 6. (Color online) Simulated squared wave functions of the exciton component as a function of radius, for three selected lower polariton states. Polar numbers $(m, n)$ are indicated. Thin curve: lower polariton confinement potential, computed from the photon and exciton potentials (this latter being the zero energy reference). The curves are offset by the corresponding eigenenergies.

pattern formed by the extended lower polariton branch, which is particularly evident in the 9 and $19 \mu \mathrm{m}$ spectra. The spatial fluctuations of the PL peak-energy and the varying intensity suggest the occurrence of weak spatial localization over a few tens of $\mu \mathrm{m}$. This polariton localization is indeed expected in planar MCs, due to the intrinsic fluctuations of the cavity thickness produced in epitaxial growth. ${ }^{25}$

The measured spatial pattern can be simulated using the model of the previous section. As an example, we plot in Fig. 6 the square of the radial exciton wave functions for a few lower polariton modes in the case of a 8.6- $\mu \mathrm{m}$-diameter mesa, as obtained from the simulation. This wave function is normalized. It is very important to point out that the corresponding photon component (not shown) has practically identical shape. This confirms that photon confinement is simply produced by the photonic structure, while the exciton confinement follows from strong coupling into polariton states. For the first two confined modes, the wave function is nonzero only within the potential well. To extended polariton modes, instead, correspond exciton and photon wave functions extending over the barrier, as expected. A similar result (not shown) is found for upper polariton states. The curves in Fig. 6 should be compared to the spatial pattern displayed in Fig. 3(b) for the $9 \mu \mathrm{m}$ mesa. Though only qualitative, the match between theory and experiment is very satisfactory, particularly with respect to the shape of the extended mode showing a smaller amplitude in correspondence of the mesa.

The intensity emitted from each polariton level is proportional to the corresponding polariton population. ${ }^{9}$ Figures 2(a)-2(c) clearly show that the polariton population builds up in the lowest lying energy levels if the excitation spot is smaller than the mesa diameter, indicating a rather effective energy-relaxation mechanism towards the bottom of the trap. 


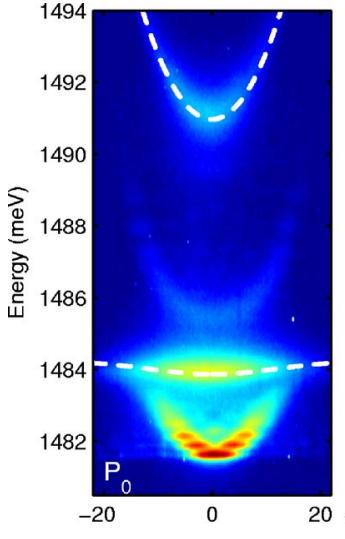

(a)

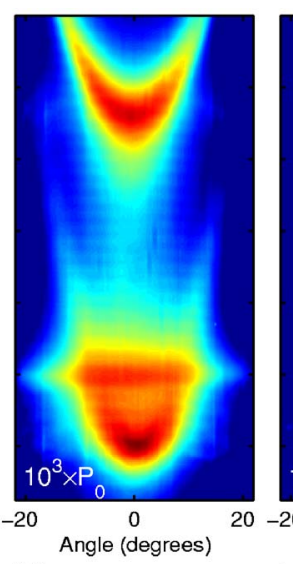

(b)

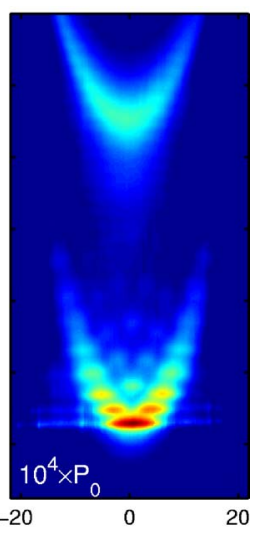

(c)
FIG. 7. (Color online) (a) Measured PL intensity for the $9 \mu \mathrm{m}$ mesa at low pump intensity $P_{0}$ in the linear regime [same data as in Fig. 2(b)]. Dashed: extended polariton dispersion from a coupled oscillator model. (b) Pump intensity $10^{3} \times P_{0}$. (c) Pump intensity $10^{4} \times P_{0}$. Log-scale covering a factor 30 (a), 30 (b), and 100 (c) from blue to red.

An analysis of the measured data indicates a Boltzmann-like distribution with a temperature of about $T=20 \mathrm{~K}$ in most measurements. This can be traced back to the presence of a large density of spatially extended states at energies above the confined states, as confirmed by the real-space measurements in Figs. 3(a)-3(c). In particular, the lower extended polariton branch (lower dashed line in Fig. 2) is almost fully excitonlike, with a vanishing photon component that results in a very long radiative lifetime. These states act as a reservoir from which polaritons relax into the confined states at lower energy. The relaxation can take place through interaction with the thermal bath of phonons ${ }^{26}$ with free carriers, ${ }^{27}$ or via mutual polariton interaction. ${ }^{28}$ The broken translational symmetry of the confined system lifts the constraint of momentum conservation, thus enhancing the relaxation efficiency compared to the case of a planar microcavity.

Finally, we have studied the PL for increasing excitation intensity. In Fig. 7(a) we plot again the PL intensity, as a function of energy and momentum, in the linear emission regime (pump intensity $P_{0}$ ) for the $9 \mu \mathrm{m}$ mesa. In Fig. 7(b) the pump intensity is 1000 times larger. Here, we observe broader spectral lines and the disappearing of strong coupling, displayed as a crossing $\left(\right.$ at $\pm 13^{\circ}$ ) between the bare exciton and cavitylike dispersions. Both features are expected as a result of the density-dependent oscillator strength saturation and the collisional broadening of the exciton transition. $^{29}$ At still higher pump intensity [Fig. 7(c)] the bound exciton spectral signature vanishes, the lasing threshold is reached, and sharp emission lines through the bare electromagnetic modes of the mesa structure appear. We point out that the linear regime of strongly coupled polaritons is preserved in this sample over two decades of pump intensity. No evidence of final-state stimulation with macroscopic occupation of the ground polariton level was ob- served, presumably due to the low saturation density of this single-well GaAs-based sample in which exciton bleaching takes place before polariton bosonic stimulation. Samples with more than one QW or based on II-VI semiconductors ${ }^{17}$ should instead make it possible to achieve polariton BoseEinstein condensation in these traps. ${ }^{16}$

\section{CONCLUSIONS}

In conclusion, we have succeeded in tailoring semiconductor microcavities in a way allowing one to obtain spatial trapping of polaritons. The traps were produced by etching a shallow mesa pattern on top of the cavity layer, before the growth of the upper mirror. Contrarily to micropillars, this technique produces a shallow confinement energy barrier, above which extended polariton states exist. Both angleresolved and spatially resolved PL demonstrate the high quality of the trapping and the simultaneous presence of extended states at higher energy, with enhanced energyrelaxation efficiency. We have developed a model for describing the polariton states in these structures, based on the solution of Maxwell equations in a perturbed planar geometry and on the linear excitonradiation coupling. The comparison between the measured spectral patterns and the simulated spectra density shows an excellent agreement, proving the effectiveness of this kind of structure.

The physics of the present system profoundly differs from the recently achieved strong coupling of a single quantum dot in a nanoresonator. ${ }^{30-32}$ In that case, the strong coupling is a direct consequence of the three-dimensional photon confinement, and produces a single pair of mixed two-level states. Here, we produce zero-dimensional trapping of polariton quasiparticles which are already in the strong coupling regime without trapping. Hence several confined and extended polariton states coexist which, due to their bosonic nature, can be occupied by more than one excitation quantum. All these features should help reaching the ideal situation of a weakly interacting cold Bose gas with a discrete energy spectrum, for which quantum collective phenomena are expected. ${ }^{14-17}$ On the other hand, the shallow confining potential makes it possible to design structures with two or more resonant traps having a significant tunneling probability. This, joined to spatially resolved resonant excitation and detection, and to the preparation of nonclassical states via parametric polariton scattering, ${ }^{12}$ can lead to a variety of easily accessible schemes for coherent manipulation of the polariton quantum phase, thus opening the way to applications in quantum information technology.

\section{ACKNOWLEDGMENTS}

This work was supported by the Quantum Photonics NCCR and Project No. 620-066060 of the Swiss National Research Foundation. We thank C. Ciuti, A. Quattropani, and P. Schwendimann for helpful discussions. We are particularly grateful to W. Langbein for enlightening advice and H.-J. Bühlmann for technical help. 
*Electronic address: vincenzo.savona@epfl.ch

${ }^{1}$ H. F. Hess, E. Betzig, T. D. Harris, L. N. Pfeiffer, and K. W. West, Science 264, 1740 (1994).

${ }^{2}$ A. P. Alivisatos, Science 271, 933 (1996).

${ }^{3}$ D. Bimberg, M. Grundmann, and N. N. Ledentsov, Quantum Dot Heterostructures (Wiley, New York, 1999).

${ }^{4}$ A. Hartmann, Y. Ducommun, E. Kapon, U. Hohenester, and E. Molinari, Phys. Rev. Lett. 84, 5648 (2000).

${ }^{5}$ E. Biolatti, R. C. Iotti, P. Zanardi, and F. Rossi, Phys. Rev. Lett. 85, 5647 (2000).

${ }^{6}$ C. Weisbuch, M. Nishioka, A. Ishikawa, and Y. Arakawa, Phys. Rev. Lett. 69, 3314 (1992).

${ }^{7}$ R. Houdré, C. Weisbuch, R. P. Stanley, U. Oesterle, P. Pellandini, and M. Ilegems, Phys. Rev. Lett. 73, 2043 (1994).

${ }^{8}$ V. Savona, L. C. Andreani, P. Schwendimann, and A. Quattropani, Solid State Commun. 93, 733 (1995).

${ }^{9}$ V. Savona, F. Tassone, C. Piermarocchi, P. Schwendimann, and A. Quattropani, Phys. Rev. B 53, 13051 (1996).

${ }^{10}$ R. M. Stevenson, V. N. Astratov, M. S. Skolnick, D. M. Whittaker, M. Emam-Ismail, A. I. Tartakovskii, P. G. Savvidis, J. J. Baumberg, and J. S. Roberts, Phys. Rev. Lett. 85, 3680 (2000).

${ }^{11}$ W. Langbein, Phys. Rev. B 70, 205301 (2004).

${ }^{12}$ S. Savasta, O. Di Stefano, V. Savona, and W. Langbein, Phys. Rev. Lett. 94, 246401 (2005).

${ }^{13}$ J. P. Karr, A. Baas, R. Houdré, and E. Giacobino, Phys. Rev. A 69, 031802(R) (2004).

${ }^{14}$ J. Lauwers, A. Verbeure, and V. A. Zagrebnov, J. Phys. A 36, L169 (2003).

${ }^{15}$ D. Snoke, Science 298, 1368 (2002).

${ }^{16}$ V. Savona and D. Sarchi, Phys. Status Solidi B 242, 2290 (2005).

${ }^{17}$ J. Kasprzak, M. Richard, S. Kundermann, A. Baas, P. Jeambrun, J. M. J. Keeling, F. M. Marchetti, M. H. Szymańska, R. André, J. L. Staehli, V. Savona, P. B. Littlewood, B. Deveaud, and Le Si Dang, Nature (London) 443, 409 (2006).

${ }^{18}$ M. Bayer, T. Gutbrod, A. Forchel, T. L. Reinecke, P. A. Knipp, R.
Werner, and J. P. Reithmaier, Phys. Rev. Lett. 83, 5374 (1999).

${ }^{19}$ A. Löffler, J. P. Reithmaier, G. Sek, C. Hofmann, S. Reitzenstein, M. Kamp, and A. Forchel, Appl. Phys. Lett. 86, 111105 (2005).

${ }^{20}$ J. Bloch, R. Planel, V. Thierry-Mieg, J. M. Gérard, D. Barrier, J. Y. Marzin, and E. Costard, Superlattices Microstruct. 22, 371 (1997).

${ }^{21}$ J. Bloch, F. Boeuf, J. M. Gérard, B. Legrand, J. Y. Marzin, R. Planel, V. Thierry-Mieg, and E. Costard, Physica E (Amsterdam) 2, 915 (1998).

${ }^{22}$ G. Dasbach, M. Schwab, M. Bayer, and A. Forchel, Phys. Rev. B 64, 201309(R) (2001).

${ }^{23}$ T. Gutbrod, M. Bayer, A. Forchel, J. P. Reithmaier, T. L. Reinecke, S. Rudin, and P. A. Knipp, Phys. Rev. B 57, 9950 (1998).

${ }^{24}$ O. El Daïf, A. Baas, T. Guillet, J.-P. Brantut, R. Idrissi Kaitouni, J. L. Staehli, F. Morier-Genoud, and B. Deveaud, Appl. Phys. Lett. 88, 061105 (2006).

${ }^{25}$ W. Langbein and J. M. Hvam, Phys. Rev. Lett. 88, 047401 (2002).

${ }^{26}$ F. Tassone, C. Piermarocchi, V. Savona, A. Quattropani, and P. Schwendimann, Phys. Rev. B 56, 7554 (1997).

${ }^{27}$ P. G. Lagoudakis, M. D. Martin, J. J. Baumberg, A. Qarry, E. Cohen, and L. N. Pfeiffer, Phys. Rev. Lett. 90, 206401 (2003).

${ }^{28}$ D. Porras, C. Ciuti, J. J. Baumberg, and C. Tejedor, Phys. Rev. B 66, 085304 (2002).

${ }^{29}$ G. Khitrova, H. M. Gibbs, F. Jahnke, M. Kira, and S. W. Koch, Rev. Mod. Phys. 71, 1591 (1999).

${ }^{30}$ J. P. Reithmaier, G. Sek, A. Löffler, C. Hofmann, S. Kuhn, S. Reitzenstein, L. V. Keldysh, V. D. Kulakovskii, T. L. Reinecke, and A. Forchel, Nature (London) 432, 197 (2004).

${ }^{31}$ T. Yoshie, A. Scherer, J. Hendrickson, G. Khitrova, H. M. Gibbs, G. Rupper, C. Ell, O. B. Shchekin, and D. G. Deppe, Nature (London) 432, 200 (2004).

${ }^{32}$ E. Peter, P. Senellart, D. Martrou, A. Lemaître, J. Hours, J. M. Gérard, and J. Bloch, Phys. Rev. Lett. 95, 067401 (2005). 